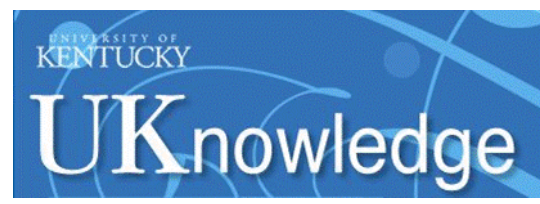

University of Kentucky

UKnowledge

9-12-2017

\title{
The Carbon and Nitrogen Abundance Ratio in the Broad Line Region of Tidal Disruption Events
}

\author{
Chenwei Yang \\ University of Science and Technology of China, China \\ Tinggui Wang \\ University of Science and Technology of China, China \\ Gary J. Ferland \\ University of Kentucky, gary@uky.edu \\ Liming Dou \\ Guangzhou University, China \\ Hongyan Zhou \\ Polar Research Institute of China, China
}

See next page for additional authors

Follow this and additional works at: https://uknowledge.uky.edu/physastron_facpub

Part of the Astrophysics and Astronomy Commons, and the Physics Commons

Right click to open a feedback form in a new tab to let us know how this document benefits you.

\section{Repository Citation}

Yang, Chenwei; Wang, Tinggui; Ferland, Gary J.; Dou, Liming; Zhou, Hongyan; Jiang, Ning; and Sheng, Zhenfeng, "The Carbon and Nitrogen Abundance Ratio in the Broad Line Region of Tidal Disruption Events" (2017). Physics and Astronomy Faculty Publications. 488.

https://uknowledge.uky.edu/physastron_facpub/488

This Article is brought to you for free and open access by the Physics and Astronomy at UKnowledge. It has been accepted for inclusion in Physics and Astronomy Faculty Publications by an authorized administrator of UKnowledge. For more information, please contact UKnowledge@lsv.uky.edu. 


\section{The Carbon and Nitrogen Abundance Ratio in the Broad Line Region of Tidal Disruption Events}

Digital Object Identifier (DOI)

https://doi.org/10.3847/1538-4357/aa8598

Notes/Citation Information

Published in The Astrophysical Journal, v. 846, no. 2, 150, p. 1-8.

(C) 2017. The American Astronomical Society. All rights reserved.

The copyright holder has granted the permission for posting the article here.

Authors

Chenwei Yang, Tinggui Wang, Gary J. Ferland, Liming Dou, Hongyan Zhou, Ning Jiang, and Zhenfeng Sheng 


\title{
The Carbon and Nitrogen Abundance Ratio in the Broad Line Region of Tidal Disruption Events
}

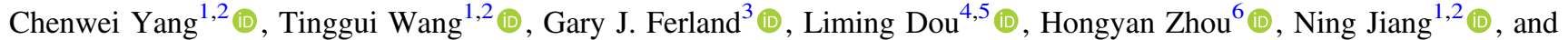 \\ Zhenfeng Sheng ${ }^{1,2}$ (1) \\ ${ }^{1}$ CAS Key Laboratory for Researches in Galaxies and Cosmology, Department of Astronomy, University of Science and Technology of China, Hefei, Anhui 230026, \\ China; cwya@ustc.edu.cn \\ ${ }^{2}$ School of Astronomy and Space Science, University of Science and Technology of China, Hefei 230026, China \\ ${ }^{3}$ Department of Physics and Astronomy, University of Kentucky, Lexington, KY 40506, USA \\ ${ }^{4}$ Center for Astrophysics, Guangzhou University, Guangzhou 510006, China \\ ${ }^{5}$ Astronomy Science and Technology Research Laboratory of Department of Education of Guangdong Province, Guangzhou 510006, China \\ ${ }^{6}$ Polar Research Institute of China, 451 Jinqiao Road, Shanghai 200136, China \\ Received 2017 June 12; revised 2017 July 28; accepted 2017 August 8; published 2017 September 12
}

\begin{abstract}
The rest-frame UV spectra of three recent tidal disruption events (TDEs), ASASSN-14li, PTF15af, and iPTF16fnl, display strong nitrogen emission lines but weak or undetectable carbon lines. In these three objects, the upper limits of the C III] $\lambda 1908 / \mathrm{N} \mathrm{III]} \lambda 1750$ ratio are about two orders of magnitude lower than those of quasars, suggesting a high abundance ratio of $[\mathrm{N} / \mathrm{C}]$. With detailed photoionization simulations, we demonstrate that $\mathrm{C}^{2+}$ and $\mathrm{N}^{2+}$ are formed in the same zone, so the $\mathrm{CIII} / \mathrm{N}$ III] ratio depends only moderately on the physical conditions in the gas and weakly on the shape of the ionizing continuum. There are smaller than 0.5 dex variations in the line ratio over wide ranges of gas densities and ionization parameters at a given metallicity. This allows a robust estimate of the relative abundance ratio of nitrogen to carbon. We derive a relative abundance ratio of $[\mathrm{N} / \mathrm{C}]>1.5$ for ASASSN-14li, and an even higher one for PTF15af and iPTF16fnl. This suggests that the broad line region in those TDE sources is made of nitrogen-enhanced core material that falls back at later times. Based on stellar evolution models, the lower limit of the disrupted star should be larger than $0.6 M_{\odot}$. The chemical abundance of the line-emitting gas provides convincing evidence that the flares originate from stellar tidal disruptions. The coincidence of the weakness of the X-ray emission with the strong broad absorption lines in PTF15af and iPTF16fnl, and the strong X-ray emission without such lines in ASASSN-li14, are analogous to quasars with and without broad absorption lines.
\end{abstract}

Key words: accretion, accretion disks - black hole physics - galaxies: abundances - galaxies: active - galaxies: nuclei - line: formation

\section{Introduction}

Evidence has been mounting that in the center of most galaxies there is a supermassive black hole. When a star passes too close (closer than the tidal radius $R_{\mathrm{T}}=r_{*}\left(M_{\mathrm{BH}} / M_{*}\right)^{1 / 3}$ ) to the supermassive black hole, it is torn apart by the tidal force of the hole. Such an event is called a stellar tidal disruption event (TDE). After the disruption, about half of the stellar debris is accreted onto the black hole, causing a strong flare peaking at soft X-ray to UV band (Rees 1988; Phinney 1989). The flare rises quickly on a timescale of about a month (Gezari et al. 2009, 2012; Arcavi et al. 2014), and falls in an approximate power-law form (Komossa \& Bade 1999; Gezari et al. 2015) or exponential form (Holoien et al. 2016). Up until this point, about 60 TDE or TDE candidates were detected up to a redshift of $z=0.89$ (Brown et al. 2015). Thus, the TDE is a unique probe for the presence of supermassive black holes in distant quiescent galaxies, for which the radius of influence of the black hole is too small to be resolved by current telescopes. Furthermore, the energetic TDE flare will ionize the gas and heat the dust in the ambient environment, causing variable coronal lines (Wang et al. 2012; Yang et al. 2013) and transient signatures in the infrared (Dou et al. 2016; Jiang et al. 2016; van Velzen et al. 2016; Dou et al. 2017). Monitoring such variable features could constrain the gas and dust properties in the galactic nucleus.

In many optically bright TDEs, transient broad emission lines (BELs) were also detected, and these lines vanish on timescales as short as one year (e.g., Wang et al. 2011; Gezari et al. 2012; Brown et al. 2015). It was proposed that the lines may come from strong outflows launched during the early high-accretion rate stage (Strubbe \& Quataert 2009) or from streams of tidal debris (Bogdanović et al. 2004; Guillochon et al. 2014). Although their origin is not fully understood, the emission lines and their time evolution carry important information about the physical, dynamical, and chemical properties of gas streams around the supermassive black hole. Such information is vital for understanding the formation and evolution of accretion flows and the launching of outflows during the super-Eddington phase.

Recently, the UV spectra of three TDEs, ASASSN-14li (Cenko et al. 2016), PTF15af (N. Blagorodnova 2017, in preparation), and iPTF16fnl (Brown et al. 2017) were taken with HST. To first order, their UV spectra are similar to those of quasars. But a closer look reveals that both of them display very strong nitrogen lines, while the expected carbon lines are relatively weak or absent. For comparison, even in one of the most nitrogen-rich quasars, Q0353-383, whose nitrogen is overabundant to oxygen by a factor of 15 compared to the solar value (Baldwin et al. 2003), the N IV] $\lambda 1486 / C$ IV $\lambda 1549$ and $\mathrm{N} \mathrm{III]} \lambda 1750 / \mathrm{C} \mathrm{III]} \lambda 1908$ line ratios are smaller than those in ASASSN-14li by about one order of magnitude. The cause of the anomalous line ratios is still under debate. It could be ascribed to an enhanced [N/C] abundance (Kochanek 2016a), collisional de-excitation of $\mathrm{C}$ III] $\lambda 1908$ line in a dense emission region, or certain radiation transfer effects (Cenko et al. 2016). 


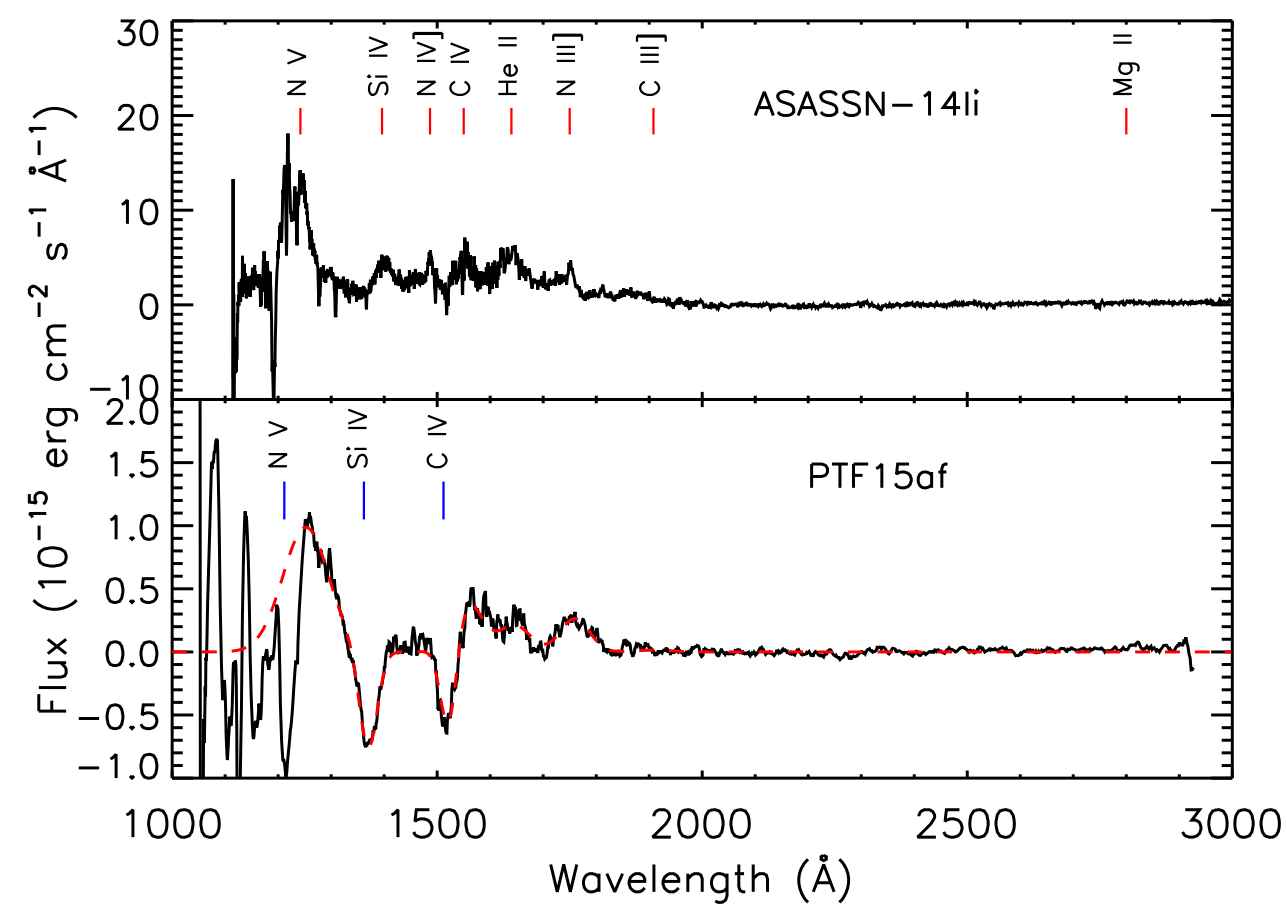

Figure 1. Upper panel: HST UV line spectrum of ASASSN-14li taken 60 days after its discovery. We have subtracted a blackbody of $3.5 \times 10^{4} \mathrm{~K}$ from the spectrum and mark the main broad emission lines with red lines. The $\mathrm{N}$ III] $\lambda 1750$ and N IV] $\lambda 1486$ lines are strong, while the $\mathrm{C}$ III] $\lambda 1908$ line is absent and the $\mathrm{C}$ IV $\lambda 1549$ line has a strength similar to the N IV] $\lambda 1486$ line. See Cenko et al. (2016) for detailed line measurements. Bottom panel: HST UV line spectrum of PTF15af after subtraction of a blackbody of $4.1 \times 10^{4} \mathrm{~K}$. We fit each emission and absorption line with one Gaussian model (red dashed line). The $\mathrm{N}$ III] $\lambda 1750$ line is strong, while the C III] $\lambda 1908$ line is absent. Strong N v $\lambda 1240, \mathrm{Si} \mathrm{IV]} \lambda 1396$, and C IV $\lambda 1549$ blueshifted broad absorption lines are also detected in the spectrum, and we mark them with blue vertical lines. The UV spectroscopic evolution of iPTF16fnl is presented in Brown et al. (2017).

Enhanced narrow [N II] emission are reported in the ultraluminous X-ray source CXOJ033831.8-352604, which could be interpreted as origins from the tidal disruption of a horizontal branch star (Irwin et al. 2010; Clausen et al. 2012).

In this work, we will use photoionization models to investigate the formation of the carbon and nitrogen lines, analyze the applicability of using the $\mathrm{C} / \mathrm{N}$ line ratio as an abundance indicator, and apply the method to ASASSN-14li. The paper is organized as follows: in Section 2, we will briefly summarize the observations of ASASSN-14li, PTF15af, and iPTF16fnl; in Section 3 we will use the numerical photoionization code CLOUDY (Ferland et al. 2013) to investigate the ionization structure and the formation of carbon and nitrogen emission lines; in Section 4 we will inspect how the line ratio changes with the gas properties and give constraint of the disrupted stars; and Section 5 is our summary.

\section{Three TDEs with UV Spectroscopy}

Recently, three TDEs, ASASSN-14li, PTF15af, and iPTF16fnl, were observed during outburst with the Space Telescope Imaging Spectrograph (STIS) on HST. The detailed multi-band evolution and optical spectroscopic analysis of ASASSN-14li and iPTF16fnl can be found in Holoien et al. (2016) and Blagorodnova et al. (2017). UV spectroscopy of ASASSN-14li and iPTF16fnl can be found in Cenko et al. (2016) and Brown et al. (2017). In this section, we will briefly introduce the UV and $\mathrm{X}$-ray observational properties of PTF15af and summarize the three objects' common features, which are relevant to our model of the three TDEs.

PTF15af was detected in the galaxy SDSS J084828.13 +220333.4 (N. Blagorodnova 2017, in preparation). A UV spectrum was taken by $H S T$ with STIS on 2015 March 8 (PI: S.
Table 1

Some Observation Feature of the Three TDEs with UV Spectroscopy

\begin{tabular}{lccc}
\hline \hline Object Name & $\log (\mathrm{C}$ III]/N III $])$ & X-Ray Strong & BAL \\
\hline ASASSN-14li & $<0.62$ & yes $^{\mathrm{a}}$ & no \\
PTF15af & $<1.21$ & no & yes \\
iPTF16fnl & $<0.97$ & no $^{\mathrm{b}}$ & yes \\
\hline
\end{tabular}

Notes.

${ }^{\text {a }}$ See Holoien et al. (2016) for X-ray evolution of ASASSN-14li.

${ }^{\mathrm{b}}$ Blagorodnova et al. (2017) find the $0.3-10 \mathrm{keV}$ luminosity of iPTF16fnl is about $2.4 \times 10^{39} \mathrm{erg} \mathrm{s}^{-1}$, four orders of magnitude lower than the peak bolometric luminosity.

Cenko), and the emission lines look quite similar to those of ASASSN-14li (Figure 1). The N V $\lambda 1240$ and N III] $\lambda 1750$ emission lines are strong, while $\mathrm{C}$ III] $\lambda 1908$ is absent. Three strong broad absorption features around $1200 \AA, 1350 \AA$ and $1500 \AA$ are identified tentatively as blueshifted N v, Si IV, and $\mathrm{C}$ IV, in analogy with broad absorption lines (BAL) quasars. The $\mathrm{N}$ IV] $\lambda 1486$ emission line falls in the BAL trough of C IV $\lambda 1549$, and the blue wing of the C IV $\lambda 1549$ emission line is also affected by the C IV $\lambda 1549 \mathrm{BAL}$, so their fluxes cannot be measured reliably. We fit the $\mathrm{N} \mathrm{III]} \lambda 1750$ line with a single Gaussian function after subtracting the continuum (see Figure 1) to get the line flux. We also derive an upper limit for C III] $\lambda 1908$ by adding a Gaussian with a width and redshift fixed at the best-fitted $\mathrm{N}$ III] $\lambda 1750$ value. The $\mathrm{N}$ III] $\lambda 1750$ emission line is at least one order of magnitude stronger than $\mathrm{C}$ III] $\lambda 1908$. Cenko et al. (2016) provided the line measurement of ASASSN-14li and we fit the C III] $\lambda 1908$ and N III] $\lambda 1750$ lines in the UV spectrum of iPTF16fnl $(t=13$ days, Figure 2 of Brown et al. 2017) with the same method as PTF15af. The 
upper limit of the line ratios of the three objects are listed in Table 1.

We also checked PTF15af's X-ray data obtained by Swift. After PTF15af was detected in the UV/optical band, Swift/ XRT took 14 pointed observations between 2015 January 27 and June 12 with a total exposure time of $\simeq 32.9 \mathrm{ksec}$ (ObsID:00033611(002-015)). We process these data following a standard data reduction procedure by the "xrtpipeline" task of the software HEAsoft (ver.6.19) with the most updated calibration files. Unfortunately, there are too few photon counts to declare an X-ray detection, even by stacking all of the observations together. Based on this fact, the estimated upper limit $0.3-10 \mathrm{keV}$ flux is $5 \times 10^{-15} \mathrm{erg} \mathrm{cm}^{-2} \mathrm{~s}^{-1}(90 \%$, assuming a simple power law with a photon index $\Gamma=2$ ). This value corresponds to an X-ray luminosity upper limit of $10^{40.9} \mathrm{erg} \mathrm{s}^{-1}$ in the $0.3-10 \mathrm{keV}$ band, making it an X-rayweak TDE.

All three TDEs display a strong N III] $\lambda 1750$ line, while the $\mathrm{C}$ III] $\lambda 1908$ line is absent. For comparison, the typical line ratio of $\mathrm{C}$ III] $\lambda 1908 / \mathrm{N} \mathrm{III]} \lambda 1750$ for quasars is $\approx 40$ (Vanden Berk et al. 2001), and in nitrogen-loud quasars the $\mathrm{C}$ III] $\lambda 1908$ line is never absent (Jiang et al. 2008). The two TDEs with weak or no detection in the X-ray (PTF15af and iPTF16fnl) display BALs, while the one with a high X-ray luminosity (ASASSN-14li) does not. We summarize the three TDEs' X-ray and BAL feature in Table 1 and discuss them in Section 4.4.

\section{Photoionization Models}

\subsection{General Considerations}

First, photoionization seems inevitable given the large luminosity in ionizing photons produced by the TDE. Furthermore, the presence of lines from a wide range of ionization levels also suggests that line-emitting gas is photoionized with a significant column density. In order to produce strong $\mathrm{C}$ IV and $\mathrm{N}$ IV lines, a substantial fraction of $\mathrm{C}$ and $\mathrm{N}$ must be in the form of $\mathrm{C}^{3+}$ or $\mathrm{N}^{3+}$. These ions are created by electrons or photons of energies above 47.9 and $47.4 \mathrm{eV}$ and destructed by electrons or photons of energies above 64.5 and $77.5 \mathrm{eV}$. If the line-emitting gas is collisionally ionized and in ionization equilibrium, the temperature will be between 0.9 and $1.8 \times 10^{5} \mathrm{~K}$, according to the equilibrium models in the CHIANTI database (Del Zanna et al. 2015). At such high temperatures, only a small fraction of $\mathrm{N}$ is in the form of $\mathrm{N}^{2+}$ due to fast collisional ionization. Therefore, the bulk of the BELs must come from photoionized gas.

Photoionization models have been employed to interpret the broad line spectra of AGN and TDE. Hamann et al. (2002) made detailed simulations for BLR in AGN and predicted several UV diagnostics of relative nitrogen abundance and overall metallicity in the gas. They adopted the AGN SED as the ionizing continuum and metal enrichment pattern for starburst galaxies. Gaskell \& Rojas Lobos (2014) simulated photoionized gas in a TDE with CLOUDY and found that radiation transfer plays an important role in determining the observed He II $\lambda 4686 / \mathrm{H} \alpha$. In some parameter regimes, they reproduced the observed very large $\mathrm{He}$ II $\lambda 4686 / \mathrm{H} \beta$ without invoking a supersolar metallicity of $\mathrm{He}$, which was proposed originally by Peterson \& Ferland (1986). Roth et al. (2016) considered a model in which both continuum and emission lines are formed in a spherical, optically thick envelope, with an optical depth to electron scattering that is very large. By considering the effect of radiation transfer, they were able to explain the large $\mathrm{He}$ II $\lambda 4686 / \mathrm{H} \alpha$ with gas at the solar abundance. However, because of the large optical depth, in their model helium is nearly fully ionized, as are metals, in order to let soft X-rays escape. Thus, it remains to be shown whether there are sufficient $\mathrm{N}^{+2}$ ions in their model to explain the observed strong $\mathrm{N}$ III] $\lambda 1750$ while the gas is still transparent to soft X-rays.

In this work we will consider models in which the lines are emitted by either debris streams or outflows illuminated by $\mathrm{UV} / \mathrm{X}$-ray radiation from TDE. Thus, the emission-line region has an open geometry with a covering factor significantly less than unity, and lines from both sides may reach the observer. For simplicity, we assume a slab geometry for the emission-line cloud. We will use CLOUDY to simulate the emission-line spectra and compare predictions with the observed line ratios to constrain the relative abundance of carbon and nitrogen.

In the condition of quasar BEL cloud, both C III] $\lambda 1908 / \mathrm{N}$ III] $\lambda 1750$ and C IV] $\lambda 1549 / \mathrm{N}$ IV] $\lambda 1486$ ratios are abundance indicators (Hamann et al. 2002). However, the N IV] $\lambda 1486$ line has a much lower critical density than the C IV $\lambda 1549$ line, so the line ratio depends strongly on gas density. Furthermore, the $\mathrm{C}$ IV $\lambda 1549$ line may be formed in much denser gas than the $\mathrm{N}$ IV] $\lambda 1486$ line. In iPTF16fnl, the N IV] $\lambda 1486$ line appears later than the C IV $\lambda 1549$ line (Brown et al. 2017), and in ASASSN14li the C IV $\lambda 1549$ and N IV] $\lambda 1486$ lines show very different profiles (Cenko et al. 2016). This evidence suggests that the bulk emission of the two lines may come from different regions. For these reasons, we will not use the C IV] $\lambda 1549 / \mathrm{N}$ IV] $\lambda 1486$ ratio as an indicator for $[\mathrm{C} / \mathrm{N}]$ to avoid uncertainties due to the possible stratification of the emission-line region. The C III] $\lambda 1908$ and N III] $\lambda 1750$ lines have similar critical densities and excitation energies, and involve ions with similar ionization energies. In Section 4.1 we will illustrate that the line ratio of $\mathrm{C} \mathrm{III]} \lambda 1908 / \mathrm{N} \mathrm{III]} \lambda 1750$ is a good indicator of $[\mathrm{C} / \mathrm{N}]$.

\subsection{The Input SED}

Multi-band follow-up observations have been carried out for ASASSN-14li since its discovery (Holoien et al. 2016). We use the observed SED 60 days after the discovery, nearly simultaneous with the UV spectroscopic observation, as the first-order approximation to the input SED. It is composed of two blackbodies: one peaks in the UV with a temperature of $3.55 \times 10^{4} \mathrm{~K}$ and a luminosity of $6.5 \times 10^{9} L_{\odot}$, and the other peaks in the soft X-ray band with a temperature of $6 \times 10^{5} \mathrm{~K}$ (or $50 \mathrm{eV}$ ) and a luminosity of about $10^{10} L_{\odot} \operatorname{erg~s}^{-1}$ (Miller et al. 2015; Holoien et al. 2016).

The ionizing continuum striking the broad line region is likely not exactly the same as the observed SED for a number of reasons. First, the SED from an TDE may be intrinsically anisotropic, and the observed SED may include reprocessed emission produced by material in or outside of the emissionline region. Second, the observed SED may be affected by the extinction of the host galaxy. Finally, if the light-crossing time of the emission-line region is comparable to the evolution timescale of the flare, the lag between the continuum and emission line should be taken into account (Wang et al. 2012; Saxton et al. 2016).

For these reasons, we first test if our results are sensitive to the shape of the input SED. We change the relative strength of the two blackbodies by a factor of four and find that $\mathrm{N}$ III] $\lambda 1750 / \mathrm{C} \mathrm{III]} \lambda 1908$ alters by less than $10 \%$ for the models 


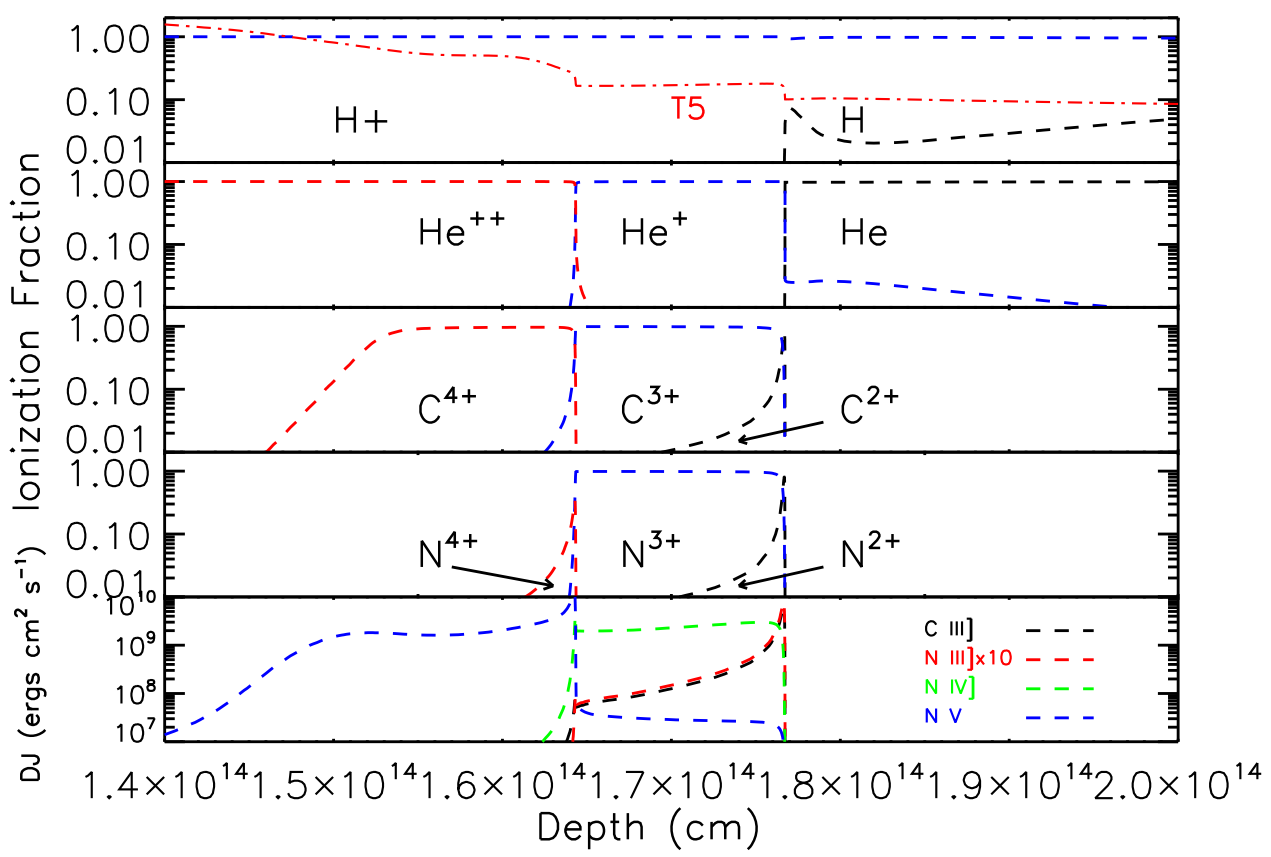

Figure 2. Ionization fractions of different elements and line emissivities multiplied by spatial depth as a function of spatial depth into the cloud in one of our models $\left(\log U=0, \log n_{\mathrm{H}}=10 \mathrm{~cm}^{-3}\right.$, solar metallicity). From top to bottom: the ionization fraction of hydrogen, helium, carbon, and nitrogen and the line emissivities of $\mathrm{C}$ III] $\lambda 1908, \mathrm{~N}$ IV] $\lambda 1486, \mathrm{~N}$ V $\lambda 1240$, and $\mathrm{N}$ III] $\lambda 1750 \times 10$. In the top panel we also plot the gas temperature in units of $10^{5} \mathrm{~K}$. The $\mathrm{N}^{3+}-\mathrm{C}^{3+}$ and $\mathrm{N}^{2+}-\mathrm{C}^{2+}$ ions reside in co-spatial zones and the line emissivities curves of $\mathrm{N} \mathrm{III]} \lambda 1750$ line match that of $\mathrm{C}$ III] $\lambda 1908$ line very well. These features are similar for other parameters.

considered in the following subsections. We also varied the temperature of each blackbody by $50 \%$, and even used a standard AGN SED as input. We found that the resultant line ratio changed by less than $20 \%$. Thus, the final results are insensitive to the detailed input SED. Therefore, for simplicity, we will only present the results for the observed SED.

\subsection{Gas Properties}

Next, we examine how the line ratio depends on the gas density $\left(n_{\mathrm{H}}\right)$, column density $\left(N_{\mathrm{H}}\right)$, dimensionless ionization parameter $\left(U=\Phi_{\mathrm{H}} / c n_{\mathrm{H}}\right.$, where $\Phi_{\mathrm{H}}$ is the photon flux at the incident surface), and the chemical composition. We run a set of plane-parallel slab models covering a large parameter range: $5<\log n_{\mathrm{H}}<11 \mathrm{~cm}^{-3}$ and $-1<\log U<1$. We do not consider higher densities $\left(n_{\mathrm{H}}>10^{11} \mathrm{~cm}^{-3}\right)$ because C III] $\lambda 1908$ and $\mathrm{N}$ III] $\lambda 1750$ lines are effectively suppressed by collisional de-excitation at those densities.

We find that when gas density is higher than $n_{\mathrm{H}}>10^{9} \mathrm{~cm}^{-3}$, the C III] $\lambda 1908$ or $\mathrm{N} \mathrm{III]} \lambda 1750$ lines become optically thick at high carbon/nitrogen abundances. In the case of a TDE, rather than static clouds, the line-emitting gas is very likely highly dynamical because the observed line profile changes with time rather rapidly (e.g., Holoien et al. 2016). In either the disk-wind or infalling gas-stream model, we expect a large internal velocity dispersion within the line-emitting gas, so we add a turbulent velocity of $100 \mathrm{~km} \mathrm{~s}^{-1}$ to make the relevant lines optically thin. This velocity is much smaller than the observed FWHMs of most of the emission lines in the UV spectra of those TDEs. As noted in Hamann et al. (2002), continuum pumping (resonant absorption of continuum photons followed by radiative decays) is insignificant for inter-combination lines with velocity dispersions smaller than $3000 \mathrm{~km} \mathrm{~s}^{-1}$.

We consider a range of gas abundances because lineemitting gas is mostly formed from the stellar debris, which is likely affected by stellar nuclear synthesis. We assume that the initial abundance of the disrupted star is similar to our Sun, although there are suggestions that the metallicity may be higher in the centers of galactic nuclei. We adopt the 2010 solar composition given by Grevesse et al. (2010), $\log (\mathrm{C} / \mathrm{H})=$ -3.57 and $\log (\mathrm{N} / \mathrm{H})=-4.17$.

As the star evolves, the $\mathrm{CN}$ cycle may gradually change the $\mathrm{C} / \mathrm{N}$ ratio in the hydrogen-burning core by converting $\mathrm{C}$ to $\mathrm{N}$ while keeping the total number of $\mathrm{C}$ and $\mathrm{N}$ the same, and finally reach a quasi-equilibrium value of $\log (\mathrm{C} / \mathrm{N}) \simeq-2.2$ in about $10^{8}$ year for a one-solar-mass star. The equilibrium ratio increases and the timescale shortens as the stellar mass increases. For stars with masses greater than 2.5 solar mass, the $\mathrm{ON}$ cycle will convert $\mathrm{O}$ into $\mathrm{N}$, increasing further the abundance of $\mathrm{N}$. In our work, we neglect the change of the other elements such as helium and oxygen, which occurs over longer timescales, and assume that all reduced carbon becomes nitrogen $(\Delta \mathrm{C} / \Delta \mathrm{N}=-1)$. Our grid of $\log \mathrm{C} / \mathrm{N}$ ranges from the solar value of 0.6 to a lower value of $-2.6([\mathrm{C} / \mathrm{N}]=$ $0 \sim-3.2$ ). Note that the tidal disruption takes place so fast that the star may not have time to mix matter located in different radii, which fall back at different times, so the debris may have a wide range of $\mathrm{C} / \mathrm{N}$ ratio across it.

The calculation stops when the neutral hydrogen fraction reaches $90 \%$. Because the lines used in the abundance diagnosis are formed in the hydrogen fully ionized zone (see below), increasing the thickness of the cloud further will not alter these line intensities. Also, when the strength of $\mathrm{N} \mathrm{III]}$ $\lambda 1750$ is comparable that of $\mathrm{N}$ IV] $\lambda 1486$, as observed, the gas must be thick enough to include the $\mathrm{N}^{2+}$ layer.

\section{Results}

\subsection{Ionization Structure and Line Emissivities}

Figure 2 illustrates the ionization structure of the gas in one of our models. This has an ionization parameter of $\log U=0$, 


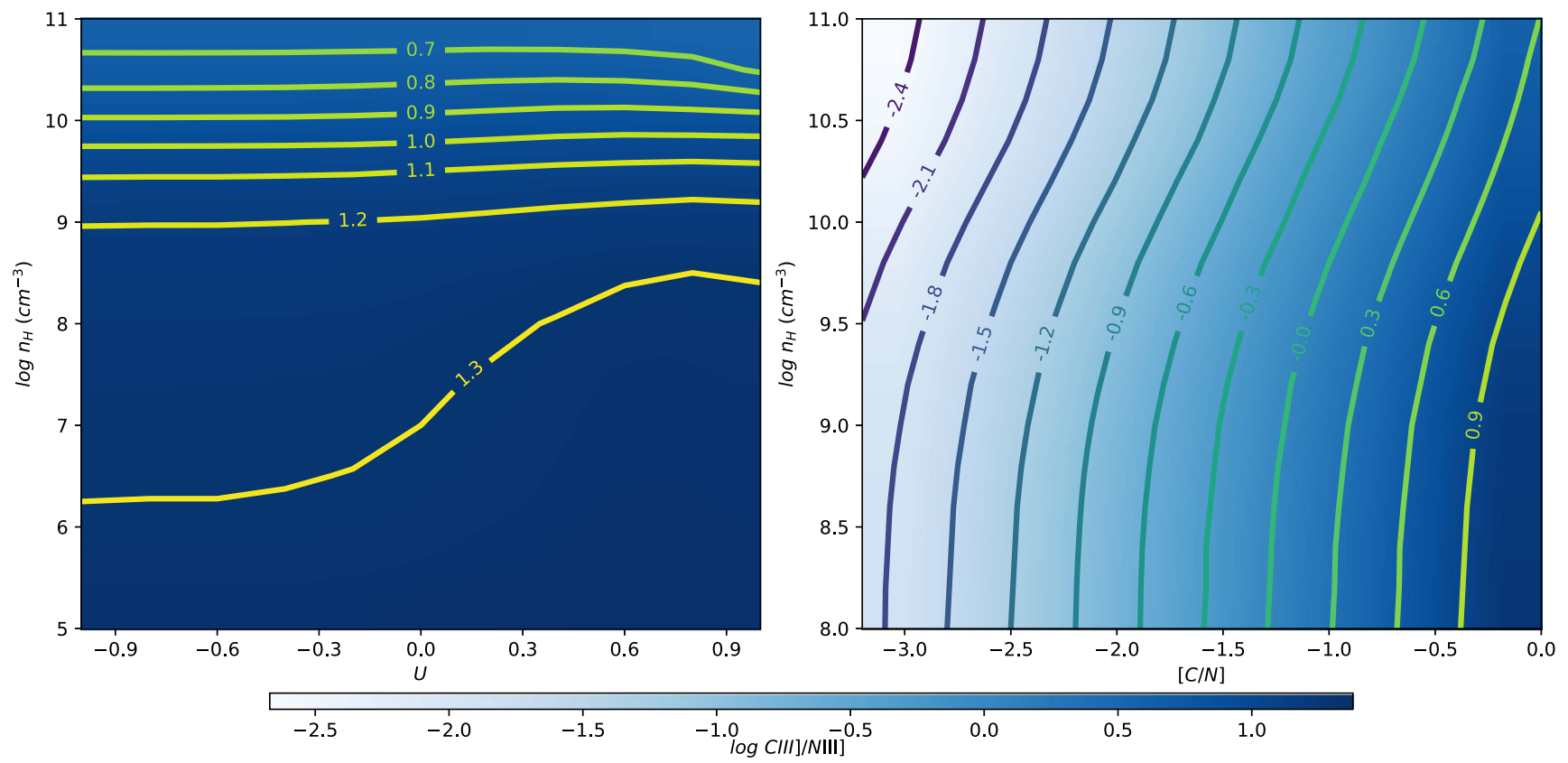

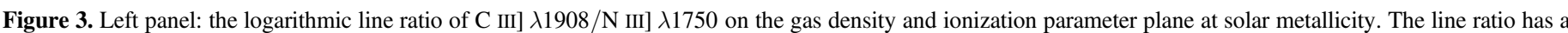

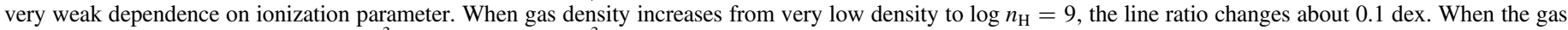

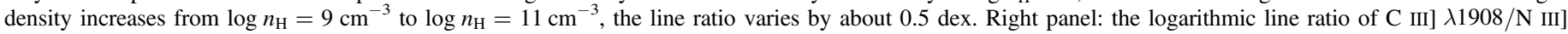
$\lambda 1750$ on the gas density and $[\mathrm{C} / \mathrm{N}]$ plane. The $\mathrm{C} \mathrm{III]} \lambda 1908 / \mathrm{N}$ III] $\lambda 1750$ ratio has much stronger dependence on $[\mathrm{C} / \mathrm{N}]$ than on gas density.

a gas density of $\log n_{\mathrm{H}}=10 \mathrm{~cm}^{-3}$, and solar metallicity. In the range of ionization parameter, gas density, and metallicity considered here, the ionization structures are similar to Figure 1 of Hamann et al. (2002). The main characteristics of the ionization structure are as follows. First, ions of $\mathrm{N}$ and $\mathrm{C}$ of the same ionization state are co-spatial. $\mathrm{N}^{4+}$ and $\mathrm{C}^{4+}$ reside within the $\mathrm{He}^{++}$zone, while $\mathrm{N}^{3+}, \mathrm{C}^{3+}$ and $\mathrm{N}^{2+}, \mathrm{C}^{2+}$ exist mainly in the $\mathrm{He}^{+}$zone. Second, the ionization fractions of $\mathrm{N}^{3+}-\mathrm{C}^{3+}$ and $\mathrm{N}^{2+}-\mathrm{C}^{2+}$ are similar at each depth, thus the absolute number densities of the pairs are proportional to their relative abundance.

Next we plot the line emissivities $J$ of $\mathrm{N} V \lambda 1240, \mathrm{~N}$ IV] $\lambda 1486, \mathrm{~N} \mathrm{III}] \lambda 1750$, and C III] $\lambda 1908$ lines in the bottom panel of Figure 2. Following Hamann et al. (2002), we multiply the emissivities $J$ by the spatial depth D to offset the tendency for the logarithmic scale. For solar abundances, the $\mathrm{N} \mathrm{III]} \lambda 1750$ line is one order of magnitude weaker than the C III] $\lambda 1908$ line, so we multiply the $\mathrm{N}$ III] $\lambda 1750$ line emissivities by a factor of 10 to make it easier to compare. We find that the profile of the two lines matches very well, and their ratio is proportional to the relative abundance (bottom panel of Figure 2). This result is reasonable because these two lines come from ions with similar ionization structure and have similar critical density and excitation energies. These results suggest that the pair C III] $\lambda 1908$ and $\mathrm{N}$ III] $\lambda 1750$ provides a good diagnostic for the abundance ratio $[\mathrm{C} / \mathrm{N}]$. In Section 4.2 we will check how their line ratio varies with other parameters, in addition to the $[\mathrm{C} / \mathrm{N}]$.

\subsection{The C III] $\lambda 1908 / \mathrm{N} \mathrm{III}] \lambda 1750$ Ratio}

In this work we consider the total emergent line fluxes produce by gas slabs. With the large simulations described in the last section, we first examine contours of the logarithmic line ratio of C III] $\lambda 1908 / \mathrm{N}$ III] $\lambda 1750$ in the ionization parameter versus the density plane (left panel of Figure 3 ). We find that at a given density and $[\mathrm{C} / \mathrm{N}]$, the line ratio depends weakly on the ionization parameter, but varies with the gas density due to slightly different critical densities for these lines. When the density is significantly lower than the critical densities $\left(n_{c} \sim 10^{9} \mathrm{~cm}^{-3}\right)$, the line ratio stays almost unchanged as the density changes. Above the critical densities, C III] $\lambda 1908 / \mathrm{N}$ III] $\lambda 1750$ decreases significantly as the density increases. When the density increases from $\log n_{\mathrm{H}}=9 \mathrm{~cm}^{-3}$ to $\log n_{\mathrm{H}}=11 \mathrm{~cm}^{-3}, \mathrm{C} \mathrm{III]} \lambda 1908 / \mathrm{N} \mathrm{III]} \lambda 1750$ varies typically by about $\sim 0.6$ dex. We also check these results for other $[\mathrm{C} / \mathrm{N}]$ values. The line ratio behaves similarly, while the values at the same density and ionization parameter roughly scale with $[\mathrm{C} / \mathrm{N}]$ value.

Since the ionization parameter has only a small effect on the line ratio, we choose $\log U=0$ as a representative value and check how the C III] $\lambda 1908 / N$ III] $\lambda 1750$ ratio varies with $[\mathrm{C} / \mathrm{N}]$. The results are plotted in the right panel of Figure 3 . We find that although the gas density affects the line ratio, the $[\mathrm{C} / \mathrm{N}]$ has a much larger effect. At a fixed gas density, when changing $[\mathrm{C} / \mathrm{N}]$ from 0 to -3.2 , the $\mathrm{C}$ III] $\lambda 1908 / \mathrm{N}$ III] $\lambda 1750$ ratio also changes by about three orders of magnitude.

In order to compare these predictions with observations, we plot the predicted line ratio of C III] $\lambda 1908 / \mathrm{N}$ III] $\lambda 1750$ as a function of $[\mathrm{C} / \mathrm{N}]$ for four densities $\log n_{\mathrm{H}}=8,9,10$, and $11 \mathrm{~cm}^{-3}$ in Figure 4. Below $\log n_{\mathrm{H}}=8$, the line ratio does not vary much with $n_{\mathrm{H}}$, while at densities higher than $10^{11} \mathrm{~cm}^{-3}$ those inter-combination lines are effectively suppressed, leaving only very weak lines. Note that at $\log n_{\mathrm{H}}=11 \mathrm{~cm}^{-3}$ and solar abundance, given the same ionizing flux $\phi_{\mathrm{H}}$ and covering factor, the line intensities of C III] $\lambda 1908$ and $N$ III] $\lambda 1750$ are only $7 \%$ and $11 \%$ of those at $\log n_{\mathrm{H}}=10$.

Figure 4 shows that the observed upper limit of $\mathrm{C}$ III] $\lambda 1908 / \mathrm{N} \mathrm{III}] \lambda 1750$ is too small to be consistent with models with solar abundance. For the density range considered here, the line ratio suggests an upper limit of $[\mathrm{C} / \mathrm{N}]<-1.2$. As we 


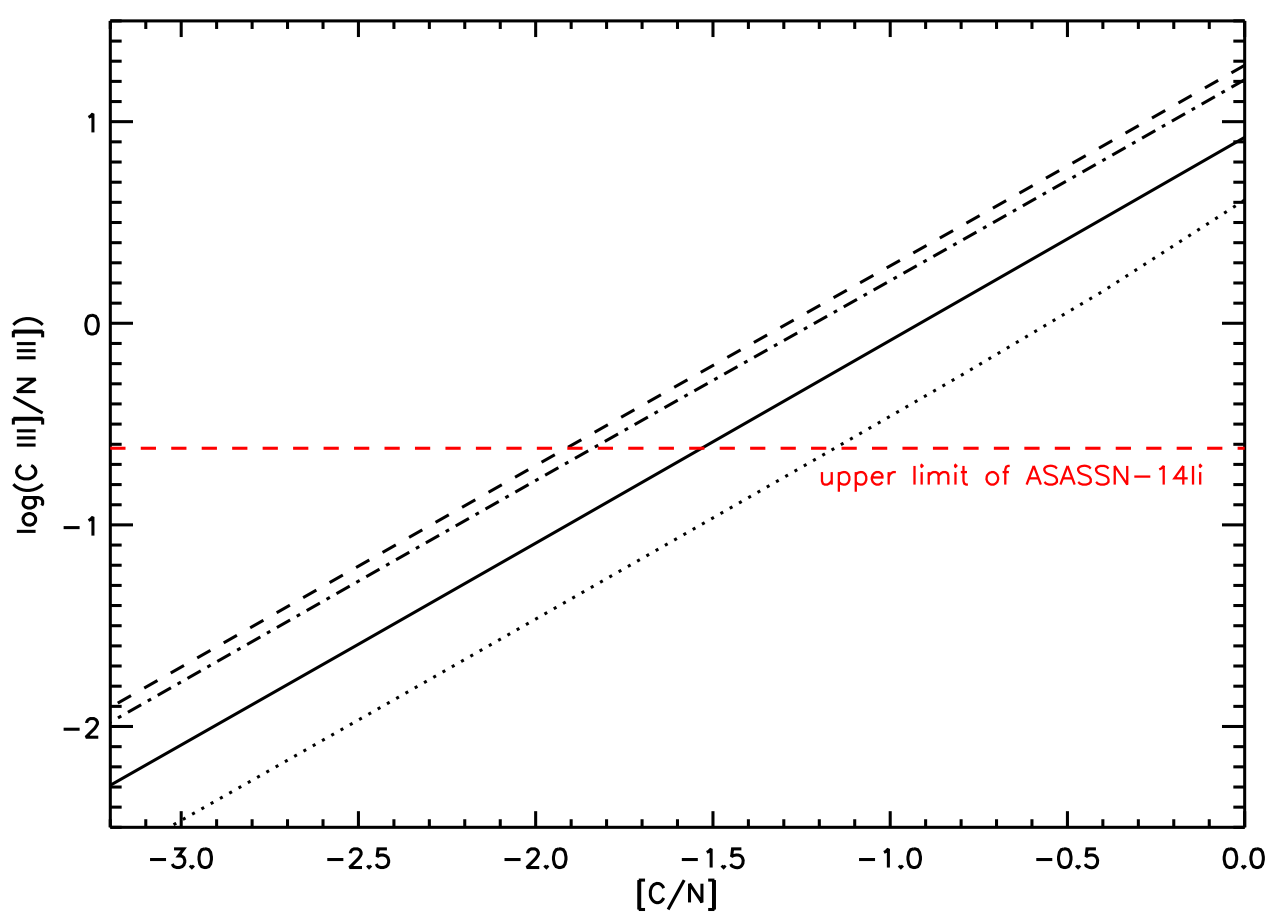

Figure 4. Predicted logarithmic line ratios of $\mathrm{C}$ III] $\lambda 1908 / \mathrm{N}$ III] $\lambda 1750$ vs. [C/N] for ionization parameter $\log U=0$. The line ratio does not depend strongly on the ionization parameter. The dashed, dashed-dotted, solid, and dotted lines represent $\log n_{\mathrm{H}}=8,9,10$ and $11 \mathrm{~cm}^{-3}$. The horizontal red dot line marks the observed upper limit line ratio of $\mathrm{C}$ III] $\lambda 1908 / \mathrm{N}$ III] $\lambda 1750$ observed in ASASSN-14li. The line ratio of C III] $\lambda 1908 / \mathrm{N}$ III] $\lambda 1750$ indicates [C/N] $<-1.5$.

have already discussed, it is likely that the line-emitting gas is distributed over a wide range in physical conditions. Because the line emissivity is proportional to $n^{2}$ until collisional excitation becomes important, the bulk of the lines should come from gas with a density close to the critical density $\left(\log n_{\mathrm{c}} \sim 10 \mathrm{~cm}^{-3}\right)$. With this assumption and using the observed line ratio from ASASSN-14li, we infer an $[\mathrm{C} / \mathrm{N}]$ of about -1.5 , which converts to a number ratio of carbon to nitrogen of $\log (\mathrm{C} / \mathrm{N})<-0.9$. We will use that value to constrain the properties of the disrupted star in the next section.

\subsection{Constraints on the Disrupted Star, and Clues about the Nature of the Line-emitting Gas}

The only three TDEs with early-stage UV spectra display a high nitrogen to carbon line ratio, suggesting that it maybe a common feature in TDEs. Based on our model, this reflects a high $\mathrm{N} / \mathrm{C}$ abundance ratio in all three TDEs. High $\mathrm{N} / \mathrm{C}$ abundance ratios of about $\log (\mathrm{C} / \mathrm{N})=-1$ have been found in several planetary nebulae (PN; Ventura et al. 2016) and a few supernovae (SNe; Fransson et al. 2005). If the line-emitting gas origins from nitrogen-enriched gas from $\mathrm{PN}$ or supernova remains, it is possible to produce high $\mathrm{N} / \mathrm{C}$ line ratios. However, unless the $\mathrm{PN}$ or $\mathrm{SNe}$ is located quite close to the black hole, it is difficult to explain how the nitrogen-enriched gas could be accreted onto the black hole without mixing with low $\mathrm{N} / \mathrm{C}$ interstellar medium which is likely massive in the galactic nucleus. So the high $\mathrm{N} / \mathrm{C}$ abundance ratio suggests that the line-emitting gas is more likely composed of material from the stellar core of the disrupted star that is affected by nuclear synthesis. As discussed in the last section, the robust upper limit of the carbon to nitrogen ratio in the line-emitting gas is $\log (\mathrm{C} / \mathrm{N})<-0.9$, so we consider that the stellar core of the disrupted star should have $\mathrm{N} / \mathrm{C} \geqslant 10$ as an essential requirement. In order to see what constraints can be put on the disrupted star and the nature of BLR, we ran a set of stellar evolution models using the code MESA (Paxton et al. 2011). We assume that the star has an initial solar metallicity of $Z=0.02$. We evolve the star from the pre-main sequence, through the main sequence, and the model calculation stops when the hydrogen in the core is less than $10^{-3}$. We do not consider the subgiant or later stages of evolution. We use the default nuclear synthesis network for hydrogen burning and conventional treatment of convection.

Figure 5 shows the fraction of the central mass with $\mathrm{N} / \mathrm{C} \geqslant 10$ as a function of stellar age for stars of masses 0.6, 0.8, 1.0, 1.2, and $1.5 M_{\odot}$. Up to $35 \%$ of the mass of the stellar core can have a high $\mathrm{C} / \mathrm{N}$ ratio during the main sequence for those stars, and the fraction decreases with stellar mass and age. The host galaxies of the three TDEs can be classified as $\mathrm{E}+\mathrm{A}$ galaxies by their strong $\mathrm{H} \delta$ absorption lines (French et al. 2016; Blagorodnova et al. 2017; Brown et al. 2017), and there is evidence that TDEs are preferentially found in E+A galaxies (Arcavi et al. 2014; French et al. 2016). If the disrupted stars were born during the last burst in an $\mathrm{E}+\mathrm{A}$ host, their ages are about $10^{8}$ year. According to Figure 5, only stars with a solar mass of 1 or above possess a core with $\mathrm{N} / \mathrm{C} \geqslant 10$. At stellar masses less than $0.6 M_{\odot}$, it requires a time longer than the age of the universe for the star to evolve to the stage where its core reaches a sufficiently high $\mathrm{N} / \mathrm{C}$ ratio. Thus, we can safely say that the disrupted star must be larger than $0.6 M_{\odot}$. In contrast, Kochanek (2016b) suggested that most disrupted stars have a mass around $0.3 M_{\odot}$. The three objects show very low C III] $\lambda 1908 / \mathrm{N}$ III] $\lambda 1750$, requiring a considerably larger stellar mass. To reconcile their results with these observations, it may be that the initial mass function (IMF) of stars around a galactic nucleus does not extend to very low stellar mass. Since we have only three cases, this may also be a coincidence. However, future UV spectroscopic observations will certainly provide important constraints on this question. 


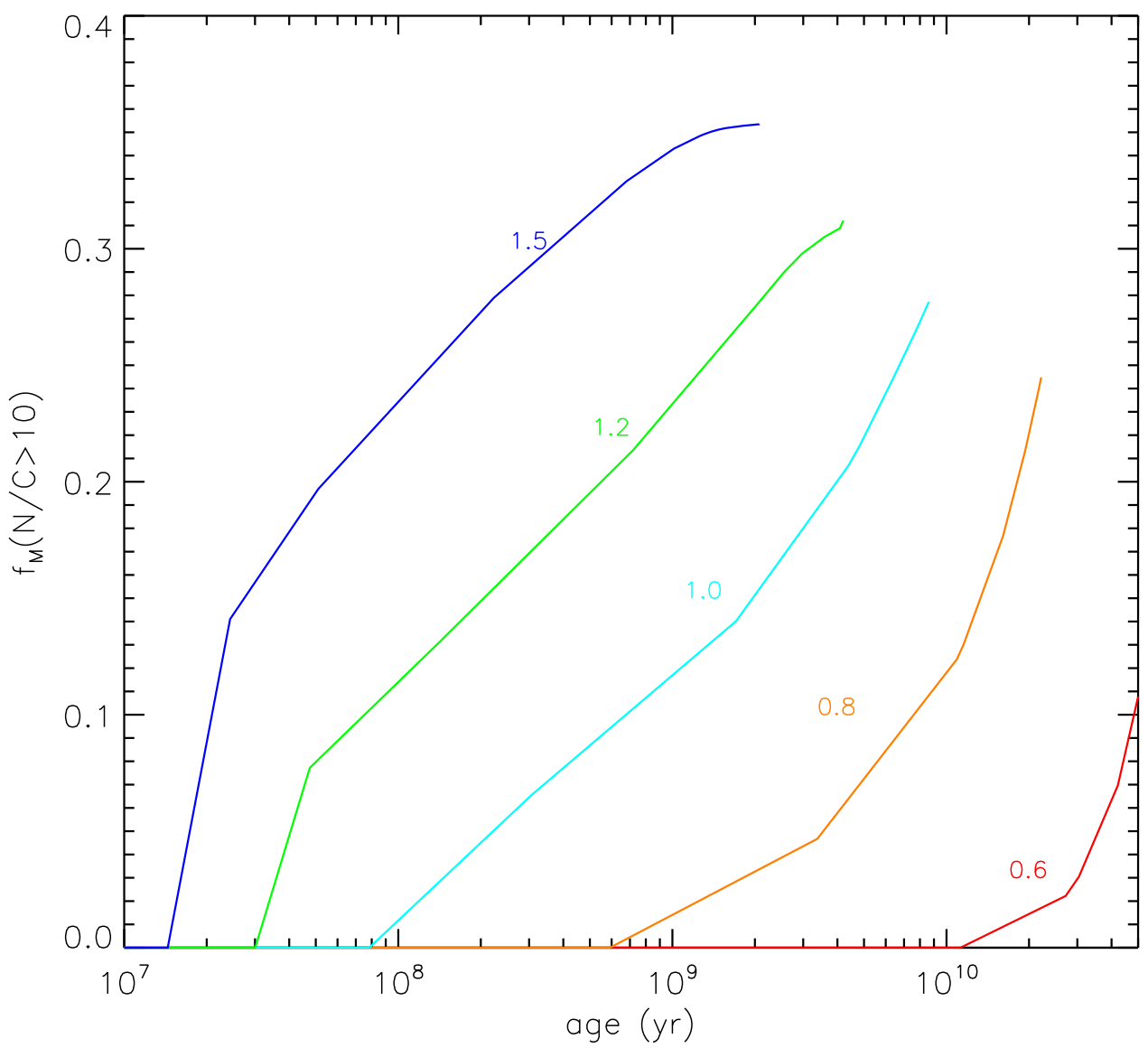

Figure 5. Fraction of the central mass with $\mathrm{N} / \mathrm{C} \geqslant 10$ as a function of stellar age for stars of masses $0.6,0.8,1.0,1.2$, and $1.5 M_{\odot}$. For stellar mass less than $0.6 M_{\odot}$, the required time is longer than the age of the universe.

When a star is disrupted, about half of the debris is bound to the black hole while the other half remains unbound. The debris from the stellar core will have an energy close to zero (e.g., Lodato \& Rossi 2011) that separates the bound and the unbound debris. Such debris eventually falls back into the black hole and forms the slowest unbound stream. For some reason, this core material makes a major contribution to the BELs, thus it must subtend a substantial solid angle relative to the continuum source. Unfortunately, theoretical work suggests that the orbits of debris align for a non-rotating black hole, so the covering factor of bound or unbounded debris will be small (Kochanek 1994). However, massive outflows are expected to launch during the early phase super-Eddington accretion (e.g., Jiang et al. 2015). We conjecture that the infalling debris may be interacting with these outflows at late times, spreading over a wide solid angle. The gas is then illuminated by the TDE flare and emits the BELs.

\subsection{Analogy to BAL Quasars}

It is known that some UV and optically discovered TDEs are $\mathrm{X}$-ray weak, although all of early discoveries of TDEs were made in the X-ray band (e.g., Grupe et al. 1995; Bade et al. 1996). With an increasing number of TDE events, there seems to be two distinct populations with regard to their X-ray properties: X-ray strong and X-ray weak. The continuum and emission-line properties for the two populations look remarkably similar, suggesting that the central engine is the same.
PTF15af and iPTF16fnl are X-ray weak and their UV spectra show BALs, while ASASSN-14li is X-ray bright and its UV spectrum does not show BALs. We note that Mg II and Fe II BALs were reported very recently in the TDE candidate PS16dtm with a NLS1-like spectrum, and it is also X-ray weak (Blanchard et al. 2017). In the literature, we found that another TDE PS1-11af also displayed Mg II BAL (Chornock et al. 2014). Unfortunately, there is no X-ray follow-up observation. Given the very small sample of TDEs, it is possible that X-ray weakness and BAL are only a coincidence. However, this relationship is remarkably similar to that observed in quasars: BAL quasars are X-ray weak, while non-BAL quasars are mostly X-ray strong. The analogy suggests that it may be real. Future spectroscopic follow-up in UV and X-ray are essential to confirm or reject this.

In quasars, it is generally accepted that BAL outflows cover only a fraction of sky. When the line of sight passes through outflows, we detect a BAL QSO, otherwise we see a non-BAL QSO. The covering factor of outflows increases with accretion rate and maybe also with the black hole mass (Ganguly et al. 2007). One argument for this is the remarkable similarity in the properties of emission lines and UV to optical continuum for BAL and non-BAL QSOs. As we have mentioned, we do not see any distinction between the emission line and the UV/ optical continuum slope between TDE with or without BALs, indicating that a similar scenario may work for TDE as well. In particular, soft X-rays are required to create $\mathrm{N}^{+4}$, so the strong $\mathrm{NV}$ emission line in those objects indicates strong soft X-ray emission out of our line of sight. 
Physically, the connection between BALs and X-ray weakness has been studied for many years in quasars, but still no consensus has been reached. It is commonly assumed that strong soft X-ray emission from quasars is filtered by a highly ionized thick shielding gas to keep the gas at the proper ionization level to ensure effective radiative acceleration (Murray et al. 1995). There is evidence for both X-ray absorption and intrinsically weak X-ray emission (Fan et al. 2009; Luo et al. 2014). Whether or not X-ray shielding is necessary for gas acceleration is also controversial (e.g., Hamann et al. 2013). It has also been proposed that the outflow itself may be highly ionized and massive, so it can produce soft X-ray absorptions (Wang et al. 2000). This may be particularly true for TDEs, which have X-ray spectra that are generally very soft, thus a rather moderately thick gas will produce strong absorption. Future UV spectroscopic monitoring of such objects will help us to understand the physical process of the formation and evolution of outflows through the study of the variation of absorption lines.

\section{Summary}

In this work we use photoionization models to calculate the C III] $\lambda 1908 / \mathrm{N}$ III] $\lambda 1750$ line ratios in the framework of a TDE. We find that reasonable ranges of the ionization parameter and SED shape have only weak effects on the line ratio. The gas density can affect line ratios by $\sim 0.5$ dex. The very small C III] $\lambda 1908 / \mathrm{N}$ III] $\lambda 1750$ line ratio can be naturally explained by the small relative abundance ratio of carbon to nitrogen. In our model calculations, the line ratio of $\mathrm{C}$ III] $\lambda 1908 / \mathrm{N}$ III] $\lambda 1750$ is a good indicator of the relative abundance of carbon to nitrogen. Using the upper limit of the line ratio observed in ASASSN-14li, we estimate the upper limit of carbon to nitrogen to be $[\mathrm{C} / \mathrm{N}]<\sim-1.5$ or $\log (\mathrm{C} / \mathrm{N})<-0.9$. BELs may come from gas that originated in a stellar core in which the CNO cycle has converted most of the carbon to nitrogen, and helium fusion has not begun to increase the carbon abundance. Based on stellar evolution models, we estimate the mass of the disrupted star to be at least $0.6 M_{\odot}$. This abnormal abundance provides strong evidence for TDEs. We also note a potential connection between the appearance of BALs and X-ray weakness in TDEs.

We acknowledge the financial support by the Fundamental Research Funds for the Central Universities, the Strategic Priority Research Program The Emergence of Cosmological Structures of the Chinese Academy of Sciences (XDB 09000000), NSFC (NSFC- 11233002, NSFC-11421303, U1431229), and National Basic Research Program of China (grant No. 2015CB857005). G.J.F. acknowledges support from NSF (1412155), NASA (ATP13-0153), and STScI (HST-AR13245, GO-12560, HST-GO-12309, GO-13310.002-A, HSTAR-13914, HST-AR-14286.001 and HST-AR-14556).

\section{ORCID iDs}

Chenwei Yang (ํ) https://orcid.org/0000-0003-4975-2433 Tinggui Wang (i) https://orcid.org/0000-0002-1517-6792 Gary J. Ferland (i) https://orcid.org/0000-0003-4503-6333 Liming Dou (1) https://orcid.org/0000-0002-4757-8622 Hongyan Zhou (i) https://orcid.org/0000-0003-1956-9021 Ning Jiang $(1)$ https://orcid.org/0000-0002-7152-3621 Zhenfeng Sheng (1) https://orcid.org/0000-0001-6938-8670

\section{References}

Alexander, K. D., Berger, E., Guillochon, J., Zauderer, B. A., \& Williams, P. K. G. 2016, ApJL, 819, L25

Arcavi, I., Gal-Yam, A., Sullivan, M., et al. 2014, ApJ, 793, 38

Bade, N., Komossa, S., \& Dahlem, M. 1996, A\&A, 309, L35

Baldwin, J. A., Hamann, F., Korista, K. T., et al. 2003, ApJ, 583, 649 Blagorodnova, N., Gezari, S., Hung, T., et al. 2017, arXiv:1703.00965

Blanchard, P. K., Nicholl, M., Berger, E., et al. 2017, arXiv:1703.07816

Bogdanović, T., Eracleous, M., Mahadevan, S., Sigurdsson, S., \& Laguna, P. 2004, ApJ, 610, 707

Brown, G. C., Levan, A. J., Stanway, E. R., et al. 2015, MNRAS, 452, 4297

Brown, J. S., Kochanek, C. S., Holoien, T. W.-S., et al. 2017, arXiv:1704. 02321

Cenko, S. B., Cucchiara, A., Roth, N., et al. 2016, ApJL, 818, L32

Chornock, R., Berger, E., Gezari, S., et al. 2014, ApJ, 780, 44

Clausen, D., Sigurdsson, S., Eracleous, M., \& Irwin, J. A. 2012, MNRAS, 424, 1268

Del Zanna, G., Dere, K. P., Young, P. R., Landi, E., \& Mason, H. E. 2015, A\&A, 582, A56

Dou, L., Wang, T., Yan, L., et al. 2017, ApJL, 841, L8

Dou, L., Wang, T.-G., Jiang, N., et al. 2016, ApJ, 832, 188

Fan, L. L., Wang, H. Y., Wang, T., et al. 2009, ApJ, 690, 1006

Ferland, G. J., Porter, R. L., van Hoof, P. A. M., et al. 2013, RMxAA, 49, 137

Fransson, C., Challis, P. M., Chevalier, R. A., et al. 2005, ApJ, 622, 991

French, K. D., Arcavi, I., \& Zabludoff, A. 2016, ApJL, 818, L21

Ganguly, R., Brotherton, M. S., Cales, S., et al. 2007, ApJ, 665, 990

Gaskell, C. M., \& Rojas Lobos, P. A. 2014, MNRAS, 438, L36

Gezari, S., Chornock, R., Lawrence, A., et al. 2015, ApJL, 815, L5

Gezari, S., Chornock, R., Rest, A., et al. 2012, Natur, 485, 217

Gezari, S., Heckman, T., Cenko, S. B., et al. 2009, ApJ, 698, 1367

Gezari, S., Hung, T., Blagorodnova, N., et al. 2016, ATel, 9433

Grevesse, N., Asplund, M., Sauval, A. J., \& Scott, P. 2010, AP\&SS, 328, 179

Grupe, D., Beuermann, K., Mannheim, K., et al. 1995, A\&A, 299, L5

Guillochon, J., Manukian, H., \& Ramirez-Ruiz, E. 2014, ApJ, 783, 23

Guillochon, J., McCourt, M., Chen, X., Johnson, M. D., \& Berger, E. 2016, ApJ, 822, 48

Hamann, F., Chartas, G., McGraw, S., et al. 2013, MNRAS, 435, 133

Hamann, F., Korista, K. T., Ferland, G. J., Warner, C., \& Baldwin, J. 2002, ApJ, 564, 592

Holoien, T. W.-S., Kochanek, C. S., Prieto, J. L., et al. 2016, MNRAS, 455, 2918 Irwin, J. A., Brink, T. G., Bregman, J. N., \& Roberts, T. P. 2010, ApJL, 712, L1 Jiang, L., Fan, X., \& Vestergaard, M. 2008, ApJ, 679, 962

Jiang, N., Dou, L., Wang, T., et al. 2016, ApJL, 828, L14

Jiang, Y.-F., Cantiello, M., Bildsten, L., Quataert, E., \& Blaes, O. 2015, ApJ, 813,74

Jose, J., Guo, Z., Long, F., et al. 2014, ATel, 6777

Kochanek, C. S. 1994, ApJ, 422, 508

Kochanek, C. S. 2016a, MNRAS, 458, 127

Kochanek, C. S. 2016b, MNRAS, 461, 371

Komossa, S., \& Bade, N. 1999, A\&A, 343, 775

Krolik, J., Piran, T., Svirski, G., \& Cheng, R. M. 2016, ApJ, 827, 127

Lodato, G., \& Rossi, E. M. 2011, MNRAS, 410, 359

Luo, B., Brandt, W. N., Alexander, D. M., et al. 2014, ApJ, 794, 70

Miller, J. M., Kaastra, J. S., Miller, M. C., et al. 2015, Natur, 526, 542

Murray, N., Chiang, J., Grossman, S. A., \& Voit, G. M. 1995, ApJ, 451, 498

Pasham, R. D., Cenko, S. B., Sadowski, A., et al. 2017, ApJL, 837, L30

Paxton, B., Bildsten, L., Dotter, A., et al. 2011, ApJS, 192, 3

Peterson, B. M., \& Ferland, G. J. 1986, Natur, 324, 345

Phinney, E. S. 1989, in IAU Symp. 136, The Center of the Galaxy, ed.

M. Morris (Dordrecht: Kluwer Academic Publishers), 543

Rees, M. J. 1988, Natur, 333, 523

Roth, N., Kasen, D., Guillochon, J., \& Ramirez-Ruiz, E. 2016, ApJ, 827, 3

Saxton, C. J., Perets, H. B., \& Baskin, A. 2016, arXiv:1612.08093

Strubbe, L. E., \& Quataert, E. 2009, MNRAS, 400, 2070

Vanden Berk, D. E., Richards, G. T., Bauer, A., et al. 2001, AJ, 122, 549

van Velzen, S., Mendez, A. J., Krolik, J. H., \& Gorjian, V. 2016, ApJ, 829, 19

Ventura, P., Stanghellini, L., Di Criscienzo, M., García-Hernández, D. A., \& Dell'Agli, F. 2016, MNRAS, 460, 3940

Wang, J., \& Merritt, D. 2004, ApJ, 600, 149

Wang, T. G., Brinkmann, W., Yuan, W., Wang, J. X., \& Zhou, Y. Y. 2000, ApJ, 545, 77

Wang, T.-G., Zhou, H.-Y., Komossa, S., et al. 2012, ApJ, 749, 115

Wang, T.-G., Zhou, H.-Y., Wang, L.-F., Lu, H.-L., \& Xu, D. 2011, ApJ, 740, 85

Yang, C.-W., Wang, T.-G., Ferland, G., et al. 2013, ApJ, 774, 46 